\title{
Characterization of new membrane materials by means of fouling experiments Adsorption of BSA on polyetherimide- polyvinylpyrrolidone membranes*
}

\author{
H.D.W. Roesink ${ }^{1}$, M.A.M. Beerlage, W. Potman, Th. van den Boomgaard, \\ M.H.V. Mulder and C.A. Smolders \\ University of Twente, Faculty of Chemical Technology, P.O. Box 217, 7500 AE Enschede \\ (The Netherlands)
}

(Received 17 May 1990; accepted 22 August 1990)

\begin{abstract}
The hydrophilicity of polyetherimide-polyvinylpyrrolidone (PEI-PVP) microfiltration membranes can be adjusted by means of a suitable post-treatment. The influence of the nature of the membrane surface on fouling properties was studied using permeation experiments before and after exposure to a protein (BSA) solution and adsorption experiments with ${ }^{14} \mathrm{C}$ labelled BSA. A correlation between the permeation experiments and the radiolabelled BSA adsorption experiments was found. The PVP in the membrane matrix prevents BSA adsorption taking place to a large extent and it appeared that heat-treated PEI-PVP membranes showed the same nonfouling behaviour as, for example, cellulose acetate membranes.
\end{abstract}

\section{INTRODUCTION}

Flux decline is caused by phenomena like concentration polarization, gel layer formation, pore blocking, adsorption, etc. and it is one of the most limiting factors in the acceptance of membrane separation processes like ultrafiltration and microfiltration. An extended survey of flux decline in membrane processes has been given by Van den Berg and Smolders [1]. It especially occurs for complex fluids that are used in the biotechnology and food industries. It is generally recognized that the adsorption of components of the fluids (e.g., proteins, surfactants, lipids) on membrane surfaces is a critical element in membrane fouling. Protein adsorption is a nearly universal phenomenon, so much research has been performed in the past to describe its occurrence (see,

*In Honour of Professor Dr Hans Lyklema on the occasion of his 60th Birthday, 23 November 1990.

${ }^{1}$ Present address: X-Flow, P.O. Box 545, 7500 AM Enschede, The Netherlands. 
for example, the work of Lyklema and coworkers [2,3] and Norde [4]). Proteins adsorb on membrane surfaces particularly because of electrostatic forces and/or hydrophobic interactions between certain domains in a protein molecule and the hydrophobic membrane material. A key to improved fouling resistance is the development of hydrophilic, low surface charge membranes [5], since it is generally accepted that hydrophilic materials are less sensitive to adsorption than hydrophobic ones [6-11]. Unfortunately, most inherently hydrophilic membrane materials are not resistant to high temperatures, extremes of $\mathrm{pH}$, cleaning agents, etc. Therefore, many investigations have been done to develop hydrophilic and resistant membranes. Most efforts have been aimed at rendering intrinsic, highly resistant polymer materials hydrophilic by suitable post-treatments [8]. In our research group a program has been started to develop such highly resistant, hydrophilic microfiltration and ultrafiltration membranes. The development of a microfiltration membrane has already been successful $[12,13]$. Membranes are prepared from a solution containing the highly resistant polymer polyetherimide (PEI) and the hydrophilic polymer polyvinylpyrrolidone (PVP) using the so-called phase inversion technique. One of the most important features of this development is the presence of an appreciable amount of PVP in the polymeric membrane matrix, despite the PVP being soluble in the aqueous coagulation bath. The membrane matrix material appears to be a heterogeneous blend. The PVP in the membrane matrix (up to $30 \mathrm{wt} \%$ based on total polymer) is mostly located at the outer surface of the pore walls and such membranes are hydrophilic. The explanation for this phenomenon is as follows: the membrane formation proceeds according to a liquid-liquid demixing process, separation into a polymer rich and a polymer poor phase. The solvent diffuses out of the polymer rich phase (PEI rich phase); at a certain concentration this phase will solidify and form the membrane matrix. The water soluble PVP will tend to diffuse from the polymer solution into the polymer poor phase and the water coagulation bath, but this diffusion process is slow when compared to the liquid-liquid demixing process. For this reason, there will be some remaining PVP present in the membrane matrix, but the concentration of PVP will be the highest at the interface of the polymer rich and the polymer poor phase, i.e., at the ultimate pore walls [14]. Since PVP swells considerably in contact with water, the water fluxes are very small. After a suitable post-treatment, which reduces swelling of PVP, the fluxes of the membranes are in agreement with the pore size and porosity as can be observed with the scanning electron microscope [12]. Two essentially different types of post-treatment have been developed [12]:

(1) Crosslinking of the PVP in the polymeric membrane matrix by means of a heat treatment

The PVP in the membrane pore walls is partly entrapped in a mixture of PEI and PVP. Due to the strong interaction between PEI and PVP, the PVP is very tightly bound. It appeared that by means of a simple heat treatment 
(see Ref. [12] and below) the PVP became insoluble in water and other solvents, probably by crosslinking. After a heat treatment the membranes were still hydrophilic.

(2) Removal of the PVP with a sodium hypochlorite solution

It appeared that using $\mathrm{NaOCl}$ treatment (see Ref. [12] and below) the PVP could be selectively removed from the polymeric membrane matrix. The membranes were still wettable by water, because of the fact that after the $\mathrm{NaOCl}$ treatment a certain amount of the PVP (2-7 wt\%) is still present in the membranes. As a result of this chemical treatment the nature of the membrane surface will change.

A special effect was obtained when the $\mathrm{NaOCl}$-treated membranes were heat treated. It appeared that the membranes became hydrophobic, i.e., they were not wettable by water any longer. The reason for this effect probably is a chemical modification, due to the heat treatment of the reaction product of PVP and $\mathrm{NaOCl}[12]$.

As a result of the preparation method of the membranes from a polymeric blend and the post-treatment methods developed, it is possible to obtain both hydrophobic and hydrophilic membrane surfaces. The character of the hydrophilic membrane surface can be adjusted using different types and different concentrations of hydrophilic polymers or by applying different post-treatment methods.

A method of investigating the character of the membrane materials is to perform adsorption experiments. Smolders and Van den Boomgaard [15] suggested four different types of model foulants that are typical in adsorption phenomena during membrane filtration. Two important variables are: size of the molecule and type of the intermolecular interaction (Table 1).

In this paper some preliminary results will be presented on the adsorption of bovine serum albumin (BSA) onto the newly developed PEI-PVP membranes [12]. This model foulant compound has often been used in adsorption studies, especially with respect to ultrafiltration membranes $[6,7,9,11,16-20]$. Robertson and Zydney [20] studied the BSA uptake in the different regions (skin, substructure and matrix) of asymmetric ultrafiltration membranes with pore dimensions similar to those of the protein. Their results indicate that a minority of the protein uptake is in the skin but with profound effects on the

\section{TABLE 1}

Model foulants for systematic adsorption studies using membranes [14]

Amphipolar interactions

(hydrophobic/hydrophilic)

Low molecular weight

High molecular weight surfactants

proteins
Unipolar interactions (hydrophilic)

sugar derivatives, oligomeric polyethers carbohydrate polymers, polyethers 
membrane hydraulic permeabilities, which is understandable taking into account that the thin top layer of an asymmetric membrane contains the smallest pores. By far the majority of experiments, described in the literature, have been performed by exposing the top layer of commercially available ultrafiltration membranes to the protein solution directly in the filtration setup. In this work the microfiltration membranes are exposed to the protein solution in a separate device, since due to the hydrophilic nature and the relatively large pore size of the microfiltration membranes $(0.05 \mu \mathrm{m})$, the entire porous structure would be wetted by the protein solution anyway.

The fouling behaviour of different types of PEI-PVP membranes has been investigated using permeation experiments before and after exposure to a BSA solution. In a separate experiment the amount of adsorbed BSA on the membranes has been measured using radiolabelled ${ }^{14} \mathrm{C}$ BSA.

\section{EXPERIMENTAL}

\section{Membranes}

The flat membranes were machine cast form a PEI-PVP-NMP (NMP is $N$-methyl-2-pyrrolidone) solution. The total polymer concentration was 25 wt\%, while the weight ratio PEI to PVP was 16 to 9 . According to the manufacturer the $M_{\mathrm{w}}$ of the PVP was 360000 , while from size exclusion chromatography a $M_{\mathrm{w}}$ of 423000 was found [12]. The temperature of the coagulation bath was $38^{\circ} \mathrm{C}$ and the residence time in the humid air gap was $3 \mathrm{~s}$. The pore size in the top layer of the membranes is maximally $0.05 \mu \mathrm{m}$. The weight fraction of PVP in the membranes is $20 \mathrm{wt} \%$ (based on total polymer), since a part of the PVP will diffuse into the aqueous coagulation bath. It has to be emphasized that the PVP is located mostly at the surface of the pore walls [12].

The microfiltration membranes were used untreated or they were post-treated as follows [12]:

Heat treatment: after preparation the membranes were thoroughly flushed with water. The water was removed by means of a solvent replacement technique using an ethanol/hexane liquid exchange treatment and the membranes were placed in an oven at $150^{\circ} \mathrm{C}$ for $15 \mathrm{~h}$. After this treatment, no PVP leached out anymore, even at elevated temperatures $\left(80^{\circ} \mathrm{C}\right)$ of the water bath.

$\mathrm{NaOCl}$ treatment: the membranes were thoroughly flushed after preparation and placed in $\mathrm{NaOCl}$ solution $\left(4 \mathrm{~g} \mathrm{l}^{-1}\right)$ for $48 \mathrm{~h}$. The membranes were thoroughly flushed with demineralized water before adsorption and filtration experiments. The membranes were still wettable by water, even when they were used for aqueous filtration experiments over a period of three months.

Heat treatment after $\mathrm{NaOCl}$ treatment: using an ethanol/hexane liquid exchange technique the membranes were dehydrated after the $\mathrm{NaOCl}$ treatment and placed in an oven at $150^{\circ} \mathrm{C}$ or $15 \mathrm{~h}$. Since the obtained membranes were 
hydrophobic after this treatment, ethanol was used as a wetting agent before measuring the water flux.

\section{Water pre-treatment}

The demineralized and ultrafiltered water was purified using reverse osmosis.

BSA solutions

The BSA (No A-2153, fraction V, 96-99\% albumin) was purchased from Sigma Chemical Company. The solutions of BSA were prepared in a phosphate buffer at $\mathrm{pH} 7.4 \pm 0.05$ with $5.84 \mathrm{~g} \mathrm{l}^{-1} \mathrm{NaCl}$ added, to give a solution with ionic strength $I=0.125 \mathrm{~N}$ The $\mathrm{pH}$ was adjusted in the range of 2.2-8 using a citric acid/phosphate buffer. Sodium azide $\left(0.002 \mathrm{~g} \mathrm{l}^{-1}\right)$ was added to all BSA solutions.

\section{Permeation experiments}

The pure water flux of the membranes was measured at $22^{\circ} \mathrm{C}$ in a dead-end filtration setup. The transmembrane pressure was $1.5-2$ bar. Water fluxes were measured volumetrically. The diameter of the circular membranes was $7.2 \mathrm{~cm}$. The average water flux of heat-treated membranes was $650 \mathrm{l} \mathrm{m}^{-2} \mathrm{~h}^{-1} \mathrm{bar}^{-1}$, of NaOCl-treated membranes $1600 \mathrm{l} \mathrm{m}^{-2} \mathrm{~h}^{-1} \mathrm{bar}^{-1}$ and of heat-NaOCl treated membranes $1000 \mathrm{l} \mathrm{m}^{-2} \mathrm{~h}^{-1} \mathrm{bar}^{-1}$. The deviation in the water fluxes of the membranes used was $20 \%$. NaOCl-treated membranes exhibited higher water fluxes since the PVP is removed from the polymeric membrane matrix [12]. Probably due to thermal effects the water flux decreases after an additional heat treatment.

The expressions used for the relative permeate flux, relative flux reduction and the relative resistance were the same as those used by Matthiasson [6] and Nilsson [7].

$R F=J_{1} / J_{0}$, with $R F$ as the relative permeate flux, $J_{0}$ is the permeate flux before the BSA adsorption and $J_{1}$ the permeate flux after BSA adsorption. The relative flux reduction is expressed as $1-R F$.

Membranes were characterized by measuring the pure water flux $\left(J_{0}\right)$. They were then removed from the filtration setup and placed in a BSA solution at $22^{\circ} \mathrm{C}$. After the BSA adsorption the membranes were rinsed with demineralized and ultrafiltered water for $5 \mathrm{~min}$, placed in the filtration setup and the pure water flux $\left(J_{1}\right)$ was measured again. The pure water flux of the membranes before the adsorption experiments $\left(J_{0}\right)$ was constant for at least $2 \mathrm{~h}$. the water flux after the adsorption experiments was measured after a constant value $\left(J_{1}\right)$ was reached. 


\section{Measurements with radiolabelled $B S A$}

The ${ }^{14} \mathrm{C}$ labelled BSA proteins, with a specific activity of $1.85 \mathrm{MBq} \mathrm{mg}^{-1}$, were purchased from Amersham International. The protein was labelled by reductive alkylation with ${ }^{14} \mathrm{C}$ formaldehyde. The BSA solutions were prepared in the phosphate buffer ( $\mathrm{pH} 7.4)$. Membrane samples of about $10 \mathrm{mg}\left(2.2 \mathrm{~cm}^{2}\right.$ and a thickness of $0.2 \mathrm{~mm}$ ) were brought into contact with $10 \mathrm{ml}$ of a protein solution containing a fixed ratio of unlabelled BSA and a relatively small amount of radiolabelled BSA. After a certain time the membrane samples were thoroughly flushed with the phosphate buffer and brought into contact with $20 \mathrm{ml}$ of a scintillation fluid (Aqualuma, Hicol, The Netherlands). The amount of BSA adsorbed on the membrane samples was determined by measuring the adsorbed radiolabelled BSA in a scintillation detector (LKB 1219 Rackbeta).

\section{RESULTS}

\section{Permeation experiments}

The effect of BSA adsorption on heat-treated membranes is given as a relative flux reduction versus the equilibrium BSA concentration (Fig. 1). After an initial sharp increase a kind of plateau value of the flux reduction is reached. Aimar et al. [9] found similar results for adsorption studies using BSA and IRIS 3038 (polyacrylonitrile) membranes (see Table 2). The hydraulic resistance of the membranes reached a plateau value when the concentration of the BSA solution increased up to $50 \mathrm{~g} \mathrm{l}^{-1}$. Matthiasson [6] and Matthiasson and Hallström [16] performed BSA adsorption experiments with commercially available polyamide, polysulfone and cellulose acetate membranes. They found an initial increase of the relative flux reduction for BSA concentrations in the range of $0-4 \mathrm{~g}^{-1}$ followed by a plateau value of the BSA adsorption for BSA concentrations between 4 and $100 \mathrm{~g} \mathrm{l}^{-1}$.

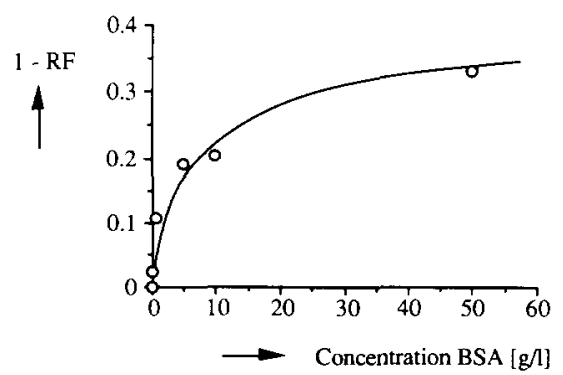

Fig. 1. Relative flux reduction $(1-R F)$ of heat-treated membranes during filtration of pure water following adsorption from a BSA solution as a function of the BSA concentration. Contact time: $16 \mathrm{~h}$. 
TABLE 2

Survey of protein adsorption studies on membranes

\begin{tabular}{llcll}
\hline $\begin{array}{l}\text { Membrane } \\
\text { material }\end{array}$ & $\begin{array}{l}\text { Starting conc. } \\
\left(\mathrm{g}^{-1}\right)\end{array}$ & $\begin{array}{l}\text { Equilibrium } \\
\text { time }\end{array}$ & Ref. & \\
\hline PA, PSF, CA & 2 & $5-60 \mathrm{~min}$ & Matthiasson and Hallström & {$[6,16]$} \\
PSF & $0.25-200$ & $5-60 \mathrm{~min}$ & Nilsson & {$[7]$} \\
RC & 0.04 & $20-40 \mathrm{~min}$ & Bornzin and Miller & {$[21]$} \\
PAN & $0.1-50$ & $5-14 \mathrm{~h}$ & Aimar et al. & {$[9]$} \\
PEI/PVP & 5 & $10-16 \mathrm{~h}$ & This paper & \\
\hline
\end{tabular}

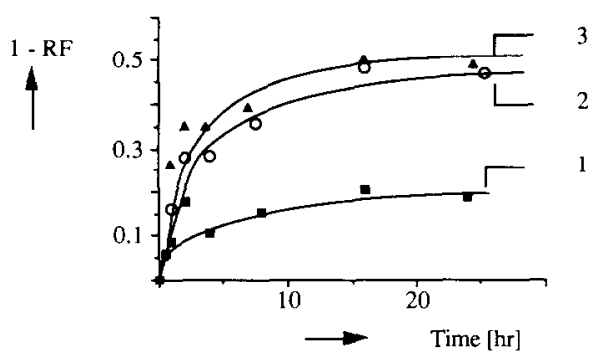

Fig. 2. Relative flux reduction $(1-R F)$ during filtration of pure water as a function of the contact time with a BSA solution. The membranes have been exposed to a BSA solution of $5 \mathrm{~g} \mathrm{l}^{-1}$. (1) heat treatment; (2) $\mathrm{NaOCl}$ treatment; (3) heat treatment after $\mathrm{NaOCl}$ treatment.

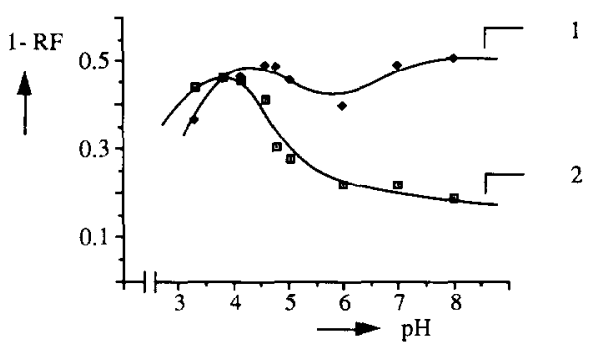

Fig. 3. Relative flux reduction $(1-R F)$ as a function of the $\mathrm{pH}$ of the BSA solution to which the membranes are exposed. The membranes were heat treated or $\mathrm{NaOCl}$ treated. RSA concentration $15 \mathrm{~g} \mathrm{l}^{-1}$. Contact time: $16 \mathrm{~h}$. (1) $\mathrm{NaOCl}$ treatment; (2) heat treatment.

The time required for the adsorption (or flux reduction) to reach an equilibrium state was determined with a BSA concentration of $5 \mathrm{~g} \mathrm{l}^{-1}$. In Fig. 2 it can be observed that after $10-16 \mathrm{~h}$ a plateau value of the flux reduction is reached. This relatively high value of the equilibrium time is in disagreement with other published results concerning adsorption kinetics (see discussion).

The influence of the $\mathrm{pH}$ on the flux reduction has also been investigated. The results are shown in Fig. 3. The $\mathrm{pH}$ dependence found for heat-treated 
membranes is in good agreement with results obtained by Matthiasson [6] and Hanemaaijer et al. [11,19]. The relative flux reduction for $\mathrm{NaOCl}$-treated membranes as a function of the $\mathrm{pH}$ is more or less constant.

\section{Adsorption experiments with radiolabelled BSA}

To compare the relative flux reduction with the amount of adsorbed BSA, adsorption experiments were performed with radiolabelled BSA. The adsorption isotherm of $\mathrm{NaOCl}$ treated membranes, presented as the amount of adsorbed BSA versus the equilibrium BSA concentration (Fig. 4), is in good agreement with the results using flux reduction experiments. The $\mathrm{NaOCl}$ treatment was used in the situation of the radiometric measurements to ensure that the amount of adsorbed BSA was high enough to be determined. The agreement is reasonable with respect to the shape of the curves and a plateau value is reached.

In Fig. 5 the same contact time $(16 \mathrm{~h})$ is found before an equilibrium value has been reached. Two different rinsing procedures were used in this work, i.e.,

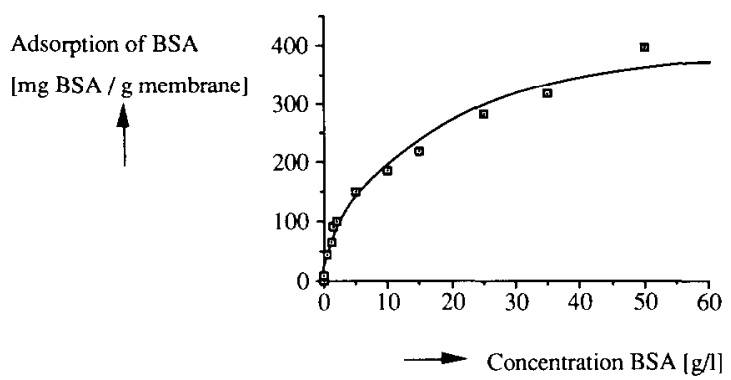

Fig. 4. Adsorption of BSA (radiometric measurement) on microfiltration membranes as a function of the BSA concentration. Contact time $16 \mathrm{~h}$. The membranes were post-treated with $\mathrm{NaOCl}$ solution.

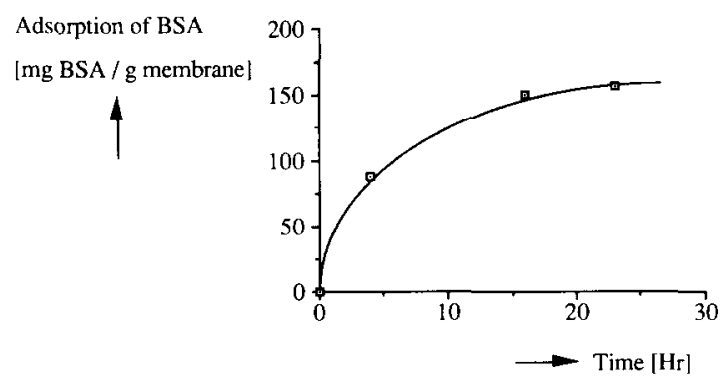

Fig. 5. Adsorption of BSA (radiometric measurement) on a microfiltration membrane as a function of the contact time. The membrane was post-treated with $\mathrm{NaOCl}$ solution. BSA concentration $5 \mathrm{~g} \mathrm{l}^{-1}$. 


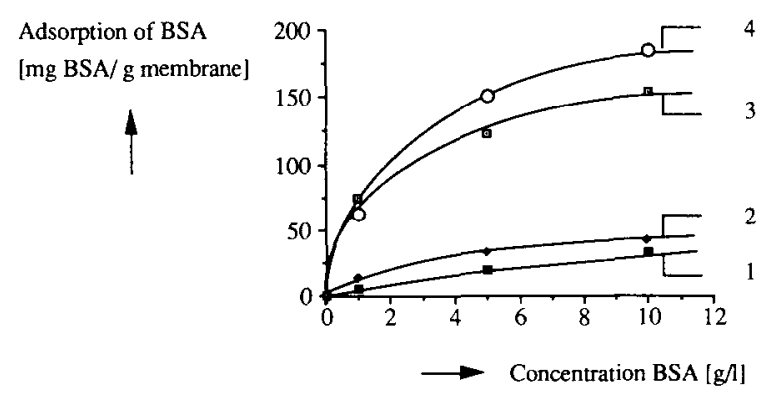

Fig. 6. Adsorption of BSA (radiometric measurement) on microfiltration membranes as a function of the concentration of BSA. The contact time was $16 \mathrm{~h}$. (1) untreated; (2) heat treatment; (3) heat treatment after $\mathrm{NaOCl}$ treatment; (4) $\mathrm{NaOCl}$ treatment.

in the permeation experiments the membranes were rinsed with pure water, while in the radiolabelled measurements phosphate buffer was used. In both case, however, the same equilibrium time was found. In fact it was very difficult to remove adsorbed BSA proteins, even when cleaning agents were used.

The adsorption isotherms for differently treated membranes presented in Fig. 6 demonstrate again very clearly that the PVP present in the heat-treated and untreated membranes reduces the adsorption of BSA. The differences between the $\mathrm{NaOCl}$-treated membranes (hydrophilic) and the hydrophobic heat$\mathrm{NaOCl}$ treated membranes is again very small. The untreated membranes exhibit the lowest BSA adsorption, but as has already been shown [12], these membranes cannot be used for permeation experiments since the PVP swells considerably. Using a cryogenic preparation technique in combination with a scanning electron microscope it was demonstrated [12] that the swollen membrane structure is still porous.

\section{DISCUSSION}

From the amount of protein adsorbed and taking into account a reasonable estimate for the membrane surface area available for adsorption a monolayer of BSA seems to be formed on the membranes that were heat treated. At higher protein concentration this will lead to a plateau value for the adsorbed amount. However a Langmuir-type equation did not fit the experimentally obtained values (or course even if the experimental isotherm did fit the Langmuir equation none of the Langmuir premises is satisfied in the case of protein adsorption). Aimar et al. [9] proposed a Freundlich equation to describe the isotherms found in their study. More experiments have to be performed to investigate whether indeed a real plateau value is reached and a second strong increase of the amount of adsorbed BSA occurs at extremely high equilibrium concentrations.

In Figs. 2 and 5 it can be observed that after $10-16 \mathrm{~h}$ a plateau value of the 
flux reduction or adsorption is reached. Matthiasson [6] performed BSA adsorption experiments (BSA concentration $2 \mathrm{~g} \mathrm{l}^{-1}$ ) using polyamide, polysulfone and cellulose acetate membranes and he found that after 5-60 min already a plateau value for the relative flux reduction was reached (see Table 2). Bornzin and Miller [21] studied the kinetics of protein adsorption and found equilibrium times between 20 and $40 \mathrm{~min}$ for BSA adsorption $\left(40 \mathrm{mg} \mathrm{l}^{-1}\right.$ ) onto Cuprophane (regenerated cellulose) surfaces. Equilibrium times in the range of 5-60 min were reported by Nilsson [7], who performed adsorption experiments with different types of proteins on polysulfone (DDS GR61 PP) ultrafiltration membranes. He found that the equilibrium time was not dependent on the concentration. Aimar et al. [9] measured the flux reduction as a function of the contact time between membrane (IRIS 3038) and BSA solutions (0.1-5.0 $\left.\mathrm{g} \mathrm{l}^{-1}\right)$. In contrast to Matthiasson and Nilsson, they found that depending on the BSA concentration, $5-14 \mathrm{~h}$ were required to reach a plateau value for the amount of adsorbed BSA. They ascribed these differences to the experimental procedure. Matthiasson [6] rinsed the membranes after adsorption experiments with saline water $(0.15 \mathrm{M} \mathrm{NaCl})$, while Aimar et al. [9] rinsed with pure distilled water, in which the solubility of BSA is very low. According to Aimar et al. it could be possible that Matthiasson dissolved some weakly bound protein in the saline water, since the amount of adsorbed BSA analysed by Aimar et al. is much larger. In this work both these rinsing procedures were used, and they both resulted in the same equilibrium time. Hence the explanation given by Aimar et al. [9] is not valid for the present work.

An important difference between the experiments of Aimar et al. [9] and Matthiasson [6] is the membrane material and it could quite well be possible that this is important for the kinetics of adsorption. Hence, more investigations concerning the kinetics of adsorption have to be performed.

The conclusion from the permeation experiments is that the fouling behaviour (i.e., adsorption behaviour as will be shown below) is strongly dependent on the kind of post-treatment of the PEI-PVP membranes. As mentioned in the introduction the membrane pore walls consist merely of PVP. This is also the case for membranes which have been heat treated only, while a $\mathrm{NaOCl}$ treatment will partly remove the PVP. From Fig. 2 then it becomes clear that the presence of PVP in the polymeric membrane reduces the adsorption of $\mathrm{BSA}$ to a large extent and consequently the flux reduction. Although the $\mathrm{NaOCl}-$ treated membranes are still fairly wettable by water and consequently hydrophilic, the flux reduction is of the same magnitude as for hydrophobic membranes. The latter type of membrane was obtained by applying a heat treatment after $\mathrm{NaOCl}$ treatment. The results indicate that the character of the hydrophilic membrane surface is very important. This effect was already reported by Defrise and Gekas [10] for microbial adhesion on microfiltration membranes.

For the heat-treated membranes, the $\mathrm{pH}$-dependence of the flux reduction 
(Fig. 3) is in good agreement with the results obtained by Matthiasson [6] and Hanemaaijer et al. [11,19]. Matthiasson performed BSA adsorption experiments with different ultrafiltration membranes and Hanemaaijer et al. found a maximum in the amount of BSA adsorbed onto polysulfone membranes at $\mathrm{pH}$ 4. Matthiasson found that the adsorption of BSA still increased at $\mathrm{pH} 4.7$ (the isoelectric point, i.e.p.) and a maximal value was reached at $\mathrm{pH}$ 3. Hanemaaijer et al. [11,19] explained the sharp increase around the i.e.p. by the tendency of the proteins to associate. It has to be stressed that the dependency of $\mathrm{pH}$ and relative flux reduction is more or less identical for hydrophobic membranes (polysulfone) and for hydrophilic membranes (cellulose acetate) $[6,11,19]$. The questionable minimum at $\mathrm{pH} 6$ for the NaOCl-treated membranes cannot be explained. Since the $\mathrm{NaOCl}$-treated membranes probably contain $\mathrm{COO}^{-}$groups [12], the surface characteristics should depend on the $\mathrm{pH}$.

The amount of adsorbed BSA correlates quite well with the relative flux reduction for the membranes after a certain post-treatment. Whether the measured amounts of radiolabelled BSA can be compared with the amount of unlabelled BSA has to be investigated. Van der Scheer et al. [22] performed experiments with mixtures of labelled and unlabelled proteins (human serum albumin, HSA) to investigate whether preferential adsorption of one of the proteins on polystyrene surfaces occurred. They found that indeed preferential adsorption of labelled HSA occurred. Van der Scheer et al. used $\mathbf{a}^{125} \mathrm{I}$ label and it can be reasoned that this label may influence the protein structure to a greater extent than the ${ }^{14} \mathrm{C}$ label does, hence in the present work no preferential adsorption of radiolabelled BSA was assumed.

It may be concluded from the adsorption isotherms (Figs 2 and 6) that the influence of the heat treatment on the chemical nature of the PVP is very small and, moreover, that the PVP is indeed located at the outer surface of the pore walls of the membranes.

As is shown in the results and the discussion, the permeation experiments and the radiometric measurements show a very clear correlation. The comparison of the amount of adsorbed BSA with experiments reported in the literature is troublesome, since the exact membrane surface area is unknown in the present work. In experiments with ultrafiltration membranes the exposed membrane surface can be used but in this work the entire porous membrane structure has been exposed to the BSA solution. A rough estimation of the specific membrane surface has been made by means of BET gas adsorption measurements. It appeared that the specific membrane surface was between 15 and $25 \mathrm{~m}^{2} \mathrm{~g}^{-1}$ of membrane. When an average membrane surface area of 20 $\mathrm{m}^{2} \mathrm{~g}^{-1}$ of membrane is taken, the amount of adsorbed BSA per $\mathrm{m}^{2}$ of the membrane surface can be calculated. Using the results of Fig. 6 the amount of adsorbed $\mathrm{BSA}$ for $\mathrm{NaOCl}$-treated membranes and heat- $\mathrm{NaOCl}$ treated membranes is about $8 \mathrm{mg} \mathrm{m}^{-2}$, while that for the heat-treated and untreated 
membranes is around $2 \mathrm{mg} \mathrm{m}^{-2}$. The latter value is in good agreement with the amount of adsorbed BSA on cellulose acetate membranes $\left(2 \mathrm{mg} \mathrm{m}^{-2}\right.$ according to Matthiasson and Hallström [16] and Bornzin and Miller [21]), while the former values are not as high as those reported for polysulfone (20 $\mathrm{mg} \mathrm{m}^{-2}$ ) and polyamide membranes $\left(30 \mathrm{mg} \mathrm{m}^{-2}\right)$ [6]. Aimar et al. [9] also reported much higher values of adsorbed amounts of BSA on IRIS 3038 membranes.

\section{CONCLUSIONS}

The presence of PVP in the polymeric membrane matrix has a positive effect on the nonfouling characteristics of the PEI-PVP membranes. Using permeation experiments it could be demonstrated that the relative flux reduction increased when the PVP from the membrane matrix was (partly) removed by means of $\mathrm{NaOCl}$ treatment. The nonfouling properties of the hydrophobic PEIPVP membranes with respect to BSA adsorption are better than those of commercially available polysulfone, polyamide and polyacrylonitrile membranes and the heat treated hydrophilic membranes exhibit the same nonfouling properties as cellulose acetate membranes. It must be stressed that the PEI-PVP membranes exhibit a much higher thermal stability and a much better chemical resistance than the cellulose acetate membranes.

The amount of adsorbed BSA on the membranes was measured using radiolabelled BSA. The results showed a good correlation with the permeation experiments and also made clear that permeation experiments, before and after adsorption, can be a very useful and a simple technique to characterize new membrane materials.

\section{ACKNOWLEDGEMENT}

The authors would like to thank G.B. van den Berg for enlightening discussions on the subject.

\section{REFERENCES}

1 G.B. van den Berg and C.A. Smolders, Filtr. Sep., March/April (1988) 115.

2 J. Lyklema, Colloids Surfaces, 10 (1984) 33.

3 W. Norde, F. MacRitchie, G. Nowicka and J. Lyklema, J. Colloid Interface Sci., 112 (1986) 447.

4 W. Norde, Adv. Colloid Interface Sci., 25 (1986) 267.

$5 \quad$ A.S. Michaels and S.L. Matson, Desalination, 53 (1985) 231.

6 E. Matthiasson, J. Membr. Sci., 16 (1983) 23.

7 J.L. Nilsson, J. Membr. Sci., 36 (1988) 147.

8 F.F. Stengaard, J. Membr. Sci., 36 (1988) 257. 
9 P. Aimar, S. Baklouti and V. Sanchez, J. Membr. Sci., 29 (1986) 207.

10 D. Defrise and V. Gekas, Process Biochem., August (1988) 105.

11 J.H. Hanemaaijer, T. Robbertsen, Th. van den Boomgaard and J.W. Gunnink, J. Membr. Sci., 40 (1989) 199.

12 H.D.W. Roesink, PhD Thesis, University of Twente, Enschede, The Netherlands, 1989.

13 H.D.W. Roesink, D.M. Koenhen, M.H.V. Mulder and C.A. Smolders, US Patent 4.798.847, 17 January 1989, assigned to X-flow B.V. Enschede, The Netherlands.

14 H.D.W. Roesink, M.J. Otto, J.A. Ronner, M.H.V. Mulder and C.A. Smolders, in preparation.

15 C.A. Smolders and Th. van den Boomgaard, J. Membr. Sci., 40 (1989) 121.

16 E. Matthiasson and B. Hallström, in B.M. McKenna, Proc. Third Int. Congr. Engineering and Food, Dublin, Ireland, September, 1983, Elsevier Applied Science, London/New York, p. 26.

17 F.F. Stengaard Desalination, 70 (1988) 207.

18 K.J. Kim, A.G. Fane an d C.J.D. Fell, Desalination, 70 (1988) 229.

19 J.H. Hanemaaijer, T. Robbertsen, Th. van den Boomgaard, C. Olieman, P. Both and D.G. Schmidt, Desalination, 68 (1988) 93.

20 B.C. Robertson and A.L. Zydney, J. Colloid Interface Sci., 134 (1990) 563.

21 G.A. Bornzin and I.F. Miller, J. Colloid Interface Sci., 86 (1982) 539.

22 A. van der Scheer, J. Feijen, J. Klein Elhorst, P.G.L.C. Krugers Dagneaux and C.A. Smolders, J. Colloid Interface Sci., 66 (1978) 136. 